\title{
Identifying HIV infection in South African women: How does a fourth generation HIV rapid test perform?
}

\author{
Authors: \\ Kapila Bhowan ${ }^{1}$ \\ Emma Kalk ${ }^{1}$ \\ Sonjiha Khan ${ }^{1}$ \\ Gayle Sherman ${ }^{1,2,3}$ \\ Affiliations: \\ ${ }^{1}$ Paediatric HIV Diagnostic \\ Syndicate, Wits Health \\ Consortium, Johannesburg \\ South Africa \\ ${ }^{2}$ Department of \\ Molecular Medicine and \\ Haematology, Faculty of \\ Health Sciences, University \\ of the Witwatersrand, \\ Johannesburg, South Africa \\ ${ }^{3}$ National Health and \\ Laboratory Service, \\ Johannesburg, South Africa \\ Correspondence to: \\ Kapila Bhowan \\ Email: \\ kbhowan@gmail.com \\ Postal address: \\ PO Box 1474, Glenvista 2058, \\ South Africa

\section{Dates:} \\ Received: 31 May 2011 \\ Accepted: 17 Nov. 2011 \\ Published: 15 Dec. 2011 \\ How to cite this article: \\ Bhowan K, Kalk E, Khan S, \\ Sherman G. Identifying HIV \\ infection in South African \\ women: How does a fourth \\ generation HIV rapid test \\ perform? Afr J Lab Med. \\ 2011;1(1), Art. \#4, 5 pages. \\ http:// dx.doi.org/10.4102/ \\ ajlm.v1i1.4
}

C) 2011. The Authors. Licensee: AOSIS OpenJournals. This work is licensed under the Creative Commons Attribution License.
Background: HIV rapid tests (RT) play an important role in tackling the HIV pandemic in South Africa. Third generation RT that detect HIV antibodies are currently used to diagnose HIV infection at the point of care. Determine Combo (DC) is the first fourth generation RT that detects both p24 antigen (p24Ag) and HIV antibodies ( $\mathrm{Ab}$ ), theoretically reducing the window period and increasing detection rates. Early detection of maternal HIV infection is important to mitigate the high risk of vertical transmission associated with acute maternal infection.

Objectives: We assessed the performance of the DC RT against third generation RT in antenatal and post-partum women.

Methods: Third generation RT Advance Quality and Acon were used in a serial algorithm to diagnose HIV infection in antenatal and post-partum women over six months at a tertiary hospital in Johannesburg, South Africa. This data provided the reference against which the DC RT was compared on plasma and whole blood samples.

Results: The 1019 participants comprised 345 (34\%) antenatal and 674 (66\%) post-partum women. Ninety women (8.8\%) tested HIV-positive of whom $59(66 \%)$ were tested antenatally, and $31(34 \%)$ post-partum yielding prevalence rates of $17.1 \%$ and $4.6 \%$ respectively. The sensitivity and specificity of the Ab component of DC on plasma antenatally was $100 \%$ $(93.8 \%-100 \%)$ and $100 \%(98.6 \%-100 \%)$ respectively and post-partum was $100 \%$ $(88.9 \%-100 \%)$ and $99.6 \%(98.8 \%-99.9 \%)$ respectively. One false positive and not a single true positive p24Ag was detected. Of 505 post-partum women who tested HIV-negative 6-12 months prior to enrolment, $12(2.4 \%)$ seroconverted.

Conclusion: The fourth generation DC offered no advantage over current third generation RT in the diagnosis of HIV infection.

\section{Introduction}

HIV rapid tests (RT) play an important role in addressing the HIV and AIDS pandemic in South Africa. They can be conducted at the point of care because they are easy to perform and require no special instrumentation. The advantage of point of care RT is that the patient can receive their HIV test result at the same clinic visit, which reduces loss to follow-up and fast tracking patients into care. ${ }^{1}$ RT are less costly than laboratory-based assays for antibody (Ab) detection namely HIV Enzyme-linked Immunosorbent Assays (ELISA) and viral detection namely HIV DNA or RNA or p24 Antigen (p24Ag).

In South Africa, pregnant women are offered counselling and testing for HIV at their first antenatal clinic visit and at 34 weeks of pregnancy if their initial HIV test was negative. ${ }^{2}$ Women at the Rahima Moosa Mother and Child Hospital (RMMCH) in Johannesburg, South Africa are also offered an HIV test immediately post-partum if their HIV status is unknown or more than six weeks have elapsed since their last negative HIV test. The importance of HIV retesting has been demonstrated by a South African study in which 3.4\% of women who tested HIV-negative at their first antenatal visit, subsequently seroconverted during pregnancy or within a year after delivery. ${ }^{3}$ The Advance Quality HIV Rapid Test (In Tec Products, Inc. Xianen, China) and Acon HIV 1/2/0 Tri-line Rapid Test (Acon Laboratories, Inc., San Diego, USA) are currently used to diagnose HIV infection in women in Prevention of Mother-to-Child transmission (PMTCT) programmes in Gauteng province, South Africa. These third generation RT detect HIV Ab that are produced in response to the virus by a serial testing algorithm as recommended by the South African PMTCT guidelines ${ }^{2}$ (Figure 1). Advance Quality is used to screen for HIV Ab and if positive, the Acon test is performed to confirm HIV status. Laboratory-based HIV ELISA and, less commonly, viral detection assays can be used as a tiebreaker to confirm an HIV status if serial RT results are discordant. ${ }^{2}$ 


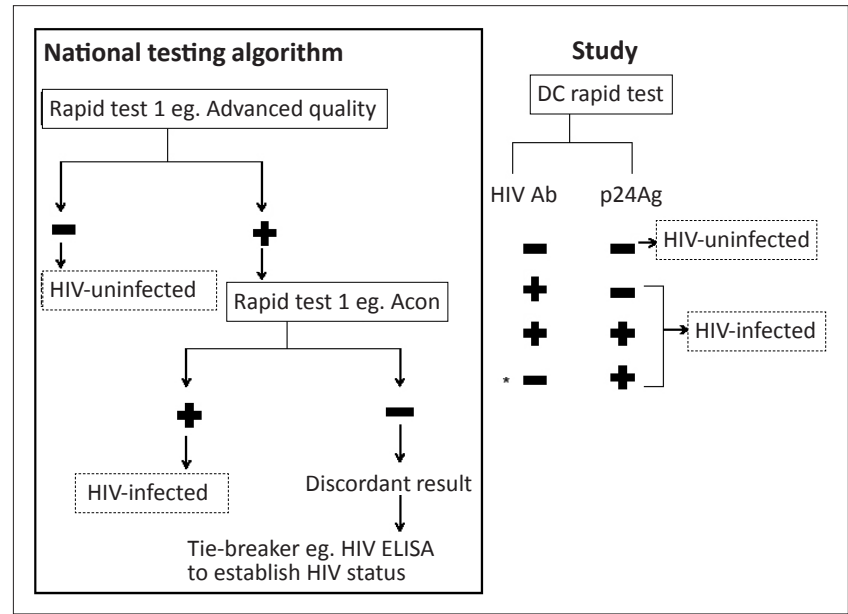

+ , positive; -, negative.

*, The combination of Determine Combo rapid test results consistent with early maternal HIV-infection were followed up with fourth generation enzyme-linked immunosorben assays (ELISA) and nucleic acid testing.

FIGURE 1: Study methodology: The Determine Combo rapid test (DC RT) was performed at the same time as the routine algorithm.

The Determine Combo HIV-1/2 Ag/Ab Combo Test (DC) (Inverness Medical, Japan Co.,Ltd) is the first fourth generation RT and can be performed on either plasma or whole blood samples. ${ }^{4}$ It is an enhancement of the third generation tests as it can detect both HIV Ab and p24Ag in a single test. ${ }^{5}$ The p24Ag is a marker of early HIV infection and is detectable in blood during the window period before HIV Ab become detectable. ${ }^{6}$ The DC RT is reported to have the potential to identify HIV infection five days (range 2-20 days) earlier than third generation RT. The reported sensitivity and specificity of the DC Ab component is 100\% (95\% confidence interval $98 \%-100 \%)$ and $100 \%$ (95\% confidence interval $98.2 \%-100 \%$ ) respectively and the sensitivity of the p24Ag component is $86.6 \%$ (95\% confidence interval $76 \%-93.7 \%){ }^{7}$

Detection of early HIV infection during the window period of third generation RT would allow more maternal HIV infections to be detected. Furthermore, during early maternal HIV infection the levels of the virus in the blood are at their peak and the risk of transmission to the infant during birth and breast-feeding is very high. ${ }^{8}$ Detection of early infection would allow more women and their infants at a high risk of vertical transmission to access PMTCT.

The performance of the fourth generation HIV DC RT in diagnosing HIV infection status antenatally and in the early post-partum period in comparison to the third generation HIV RT in routine use at public healthcare facilities in Gauteng, South Africa was assessed. The advantage offered by the DC RT over third generation RT of detecting HIV infection earlier to increase identification of women at a high risk of vertical transmission for PMTCT was investigated.

\section{Methods}

\section{Study participants}

Women attending the antenatal clinic and delivery unit at $\mathrm{RMMCH}$ in Johannesburg were invited to test for HIV infection. Counsellors interviewed the participants to establish their HIV status. Women with an unknown HIV status, those who had tested HIV-negative more than 6 weeks previously and those who reported an HIV-positive status but had no documented evidence thereof on their maternal card were invited to participate in the study. Women with a documented HIV-positive status were excluded. Written informed consent was obtained from all participants who agreed to test for HIV infection. Ethics approval (M091119) for the study was granted by the Human Research Ethics Committee at the University of the Witwatersrand, Johannesburg.

\section{Sample size}

The prevalence of HIV infection amongst antenatal women in Gauteng province, South Africa in 2008 was $29.9 \%$ (95\% confidence interval $28.4 \%-31.2 \%)^{9}$; however, the prevalence of HIV infection amongst women testing antenatally and shortly after delivery at RMMCH in 2008 was lower at $15 \%$ and $4.2 \%$ respectively since women with a known, documented HIV-positive status are excluded and proportionately more women fall into this category after delivery than antenatally. ${ }^{10}$ From RMMCH HIV testing records we expected approximately 200 women to present for testing per month with a prevalence that depended on the proportion of women testing before or after delivery. A convenience sample of all women eligible for HIV testing at $\mathrm{RMMCH}$ who agreed to participate in the study was chosen to assess the number of additional women the DC RT could identify as being infected over a 6-month period on plasma samples. For assessment of whole blood samples, the Centers for Disease Control and Prevention recommendations to include samples from at least $20 \mathrm{HIV}$-infected and $80 \mathrm{HIV}$ uninfected women were followed. ${ }^{11}$

\section{HIV testing}

Five millilitres of whole blood was drawn into an ethylenediaminetetraacetic (EDTA) tube for testing at the study site. Samples were centrifuged to obtain plasma on which the RT were performed. DC RT were performed on whole blood prior to centrifugation. All RT were performed within one hour of blood sampling by a single laboratory technician according to the manufacturer's instructions. Third generation RT Advance Quality and Acon were used serially on plasma according to the national testing algorithm ${ }^{2}$ to diagnose HIV infection (Figure 1) and were the reference standards against which the DC results were compared.

\section{Interpretation of rapid test}

The DC is a qualitative immunochromatographic test which is read visually. The test strip is divided into an HIV $\mathrm{Ab}$ window and an HIV p24Ag window. The presence of a pink line in either or both of the windows is indicative of HIV infection. Each test strip incorporates a procedural positive control and the test is considered valid only if the positive control is detected. 
The women received their RT results and post-test counselling within four hours of blood sampling. Patients that were HIV-positive on both third generation RT were referred to antiretroviral treatment clinics. The results of the DC RT were not disclosed to the patient. However, where the third generation and DC HIV Ab RT results were discordant, samples were referred for confirmatory fourth generation ELISA (ARCHITECHT ${ }^{\circledR}$ HIV Ag/Ab Combo assay; Abbott Diagnostics; Wiesbaden, Germany). Patients that had detectable p24 Ag on DC were followed up with three confirmatory tests that is viral detection assay (Vironostika HIV-1 Antigen; bioMerieux; Bosiend, The Netherlands) , viral load testing (NucliSENS EasyQ-EasyMag HIV-1, version 1.2 assay; bioMerieux; Boxtel, The Netherlands) as well as fourth generation ELISA. Disclosure of the patient's HIV status was delayed for 48 hours.

\section{Analysis}

Likelihood ratios were calculated instead of predictive values because predictive values depend on prevalence and a difference in HIV prevalence was anticipated between women tested in the antenatal and post-partum period. A positive likelihood ratio (sensitivity/(1 - specificity)) $>10$ strongly predicts HIV-infection, whereas a negative likelihood ratio $((1-$ sensitivity $) /$ specificity $)<0.1$ virtually excludes the condition.

\section{Results}

Between March and August 2010, 1019 (92.7\%) of the 1099 women eligible for HIV testing at RMMCH were enrolled in the study. Of the 1019 participants, 345 (33.9\%) were tested antenatally and $674(66.1 \%)$ post-partum. According to the routine third generation RT diagnostic algorithm, 90 (8.8\%) of the 1019 patients tested positive for HIV infection without the need to use a tiebreaker. Of the 90 HIV-infected women, $59(65.6 \%)$ were antenatal and $31(34.4 \%)$ were post-partum. The HIV prevalence amongst the women tested antenatally was $17.1 \%$ and those tested post-partum was $4.6 \%$.

Knowledge of the women's HIV status prior to undergoing HIV testing on the study was documented for those tested in the early post-partum period only (Table 1). Of the 505 women that had reported or tested HIV-negative between 6 and 12 weeks prior to study enrolment, $12(2.4 \%)$ tested positive demonstrating that new HIV infections were occurring in this population. Not all of these women had their negative HIV status documented on their maternal record and the possibility that some reported their status incorrectly cannot be excluded.

The DC RT was performed on plasma samples of the 1019 women and on whole blood samples on a subset of 380 women. All 1399 tests performed demonstrated positive control strips therefore no DC RT was invalid. Sensitivity, specificity and likelihood ratios for the DC RT were calculated separately for women who were tested antenatally and post-partum (Table 2). The sensitivity of the DC RT Ab component was 100\% in all groups of women tested irrespective of the sample type. The specificity of the DC RT Ab component in women tested post-partum was less than those tested antenatally.

The DC RT results concurred with the third generation RT results in all patients except for three post-partum women on whom both plasma and whole blood samples were tested. In two patients the $\mathrm{Ab}$ component of the DC RT was positive on both their plasma and whole blood samples but negative on both third generation RT. Laboratory based fourth generation ELISA tests on both cases were negative confirming two false positive DC HIV Ab results.

TABLE 1: HIV status of post-partum women prior to enrolment and after testing with the National testing algorithm.

\begin{tabular}{|c|c|c|c|c|c|}
\hline Reported or documented HIV status & $\begin{array}{l}\text { Time since last test } \\
\text { (weeks) }\end{array}$ & $n$ & HIV-infected & HIV-uninfected & Percentage positive \\
\hline \multirow[t]{2}{*}{ Negative } & $6-12$ & 505 & 12 & 492 & 2.4 \\
\hline & $>12$ & 107 & 3 & 104 & 2.8 \\
\hline Unknown HIV status & - & 58 & 14 & 43 & 24.1 \\
\hline Positive: reported but not documented & - & 4 & 2 & 2 & 50.0 \\
\hline Total & - & 674 & 31 & 641 & 4.6 \\
\hline
\end{tabular}

$n$, sample size.

Women of unknown HIV status had a high prevalence of HIV infection. Half of the women who reported a positive HIV status but had no documentation to substantiate a positive HIV test, tested HIV-negative.

TABLE 2: Performance of Determine Combo rapid test HIV antibody (DC RT Ab) component in plasma and whole blood samples in women tested antenatally and post-partum.

\begin{tabular}{|c|c|c|c|c|c|c|c|c|c|c|c|}
\hline $\begin{array}{l}\text { Time test was } \\
\text { conducted }\end{array}$ & Sample type & $n$ & Prevalence & $\begin{array}{l}\text { Determine } \\
\text { Combo HIV } \\
\text { Ab+/ HIV+ }\end{array}$ & Sensitivity & $\begin{array}{l}95 \% \text { confidence } \\
\text { interval }\end{array}$ & LR+ & $\begin{array}{l}\text { Determine } \\
\text { Combo HIV } \\
\text { Ab-/HIV- }\end{array}$ & Specificity & $\begin{array}{l}95 \% \text { confidence } \\
\text { interval }\end{array}$ & LR- \\
\hline \multirow[t]{2}{*}{ Antenatal } & Plasma & 345 & 17.1 & $59 / 59$ & 100 & $93.8-100.0$ & 569.2 & $286 / 286$ & 100.0 & $98.6-100.0$ & 0 \\
\hline & Whole blood & 151 & - & $59 / 59$ & 100 & $93.8-100.0$ & 184.5 & $92 / 92$ & 100.0 & 95.9-100.0 & 0 \\
\hline \multirow[t]{2}{*}{ Post-partum } & Plasma & 674 & 4.6 & $33 / 31 \dagger$ & 100 & $88.9-100.0$ & 321.5 & $641 / 643$ & 99.7 & 98.9-99.9 & 0 \\
\hline & Whole blood & 229 & - & $32 / 30 \dagger$ & 100 & $88.6-100.0$ & 99.5 & 197/199 & 99.0 & $96.4-99.7$ & 0 \\
\hline
\end{tabular}

$n$, sample size.

$\downarrow$, False positive results obtained in 2 women on both sample types. One additional women in this group tested HIV p24Ag false positive on DC. HIV+ and HIV-are HIV-infected and HIV-uninfected women respectively as defined by the third generation rapid test (RT)-algorithm.

LR+ and LR- are the positive and negative likelihood ratios.

The high positive likelihood ratios (LR+) and low negative likelihood ratios (LR-) demonstrate that the Determine Combo rapid test was a strong predictor of HIV-infection in HIV positive women and excluded HIV infection in HIV-uninfected women respectively, regardless of sample type and timing of testing. 
The DC p24Ag was reactive on plasma and the whole blood of only one patient of all 1019 women tested. This patient was suspected of having an early infection since both third generation $\mathrm{RT}$ and the $\mathrm{DC} \mathrm{Ab}$ component tested negative. However the laboratory based fourth generation HIV ELISA was negative and the viral load was undetectable indicating a false positive DC p24Ag result. No p24Ag bands on DC were obtained on any of the $90 \mathrm{HIV}$-infected women nor was a single case of early HIV infection detected.

\section{Discussion}

Considering the high enrolment rate, the sample of women tested in this study is likely to be representative of women who are tested for HIV-infection at RMMCH over half a year. Furthermore, the HIV prevalence of $17.1 \%$ and $4.6 \%$ in women testing antenatally and immediately post-partum respectively is similar to a previous description at RMMCH. ${ }^{10}$ An HIV prevalence of $24.1 \%$ in women of unknown HIV status is also comparable to the $28 \%$ prevalence previously described in 2008. However, the seroconversion rate in postpartum women of $2.4 \%$ is less than the previously described rate of $4.5 \%{ }^{10}$ The implication that new maternal infections are occurring in this population remains. In practice, a positive p24Ag DC test would require confirmation of early seroconversion by fourth generation ELISA or nucleic acid testing which may delay initiation of PMTCT. In contrast to a study that reported $16 \%$ invalid DC RT tests due to failure to detect the control, ${ }^{12}$ all DC RT in this study were valid possibly because we used fresh, not stored samples.

Sensitivity and specificity of the DC RT was comparable to plasma and whole blood in contrast to a previous report that demonstrated lower sensitivity of the DC RT in whole blood compared to serum samples. ${ }^{12}$ The sensitivity of the fourth generation DC RT in detecting HIV Ab was comparable to that of the reference third generation RT in antenatal and post-partum women in plasma and whole blood samples. The specificity of the DC RT in detecting HIV Ab was slightly reduced in whole blood and plasma in post-partum women owing to the false positive results in two women, but was still within the World Health Organization recommended range of more than $98 \% .^{13}$

The fourth generation DC RT did not detect a single true positive p24Ag, even in the $90 \mathrm{HIV}$-infected women. The sensitivity in detecting p24Ag in HIV-infected women was $0 \%$ as compared with the claim of $86.6 \%$ obtained on HIVinfected samples. ${ }^{7}$ The reason for this may be that p24Ag forms immune complexes with HIV $\mathrm{Ab}$ and thus no free p24Ag is present for detection by the DC. ${ }^{6}$ The DC RT therefore did not identify any new cases of maternal HIV infection over the 6 month study period. Possible reasons for this include that the sensitivity of the DC p24 Ag component is poor or that no women with acute HIV infection were enrolled. The former concurs with previous reports that the DC RT p24 Ag component lacks sensitivity particularly where levels of p24 Ag are below $400 \mathrm{pg} / \mathrm{mL} \cdot{ }^{12,14}$ Additionally, the p24Ag component of the DC RT has reduced sensitivity in comparison to other fourth generation viral detection assays, ${ }^{7}$ some of which are able to detect p24Ag at levels of $4 \mathrm{pg} / \mathrm{mL}$ - $5 \mathrm{pg} / \mathrm{mL} .{ }^{15}$ The initial laboratory based p24Ag assays also demonstrated poor sensitivity that was subsequently improved by denaturation of the immune complex and signal amplification to enhance p24Ag detection. ${ }^{16}$

A limitation of this study is that the incidence of HIV infection in women undergoing HIV testing at RMMCH is unknown therefore, although new maternal infections were demonstrated, it is possible that no women with acute HIV infection were enrolled during the short window period before HIV Ab and subsequent immune complex formation. Nevertheless, the DC RT did not identify any new infections over those identified by the 3rd generation RT assays over six months in the RMMCH PMTCT programme.

\section{Conclusion}

The DC RT failed to demonstrate any advantage over third generation RT currently in use in our setting in either determining HIV infection status or in identifying recently infected women. Improved sensitivity of p24 Ag detection is required before fourth generation $\mathrm{RT}$ will offer an advantage over their third generation counterparts in the field.

\section{Acknowledgements}

Funding for this study was provided by the National Health Laboratory Services. We thank all the women who participated and the clinical staff who were involved.

\section{Competing interests}

The authors declare that they have no financial or personal relationship(s) which may have inappropriately influenced them in writing this article.

\section{Authors' contributions}

All of the authors have made substantial contribution to the manuscript. G.S. (Wits Health Consortium) conceptualised and planned the study as well as performed critical revision of the manuscript. K.B. (Wits Health Consortium) analysed and interpreted the study data and prepared the manuscript. S.K. (Wits Health Consortium) performed the testing of samples, maintained quality assurance in the laboratory and performed critical revision of the manuscript. E.K. (Wits Health Consortium) assisted with patient recruitment and management in the field as well as contributed to the writing and editing of the manuscript.

\section{References}

1. Branson B. Point-of-care rapid tests for HIV antibody [homepage on the Internet] c2003 [cited 2011 Jan 02]. Available from: http://www.cdc.gov/hiv/topics/ testing/resources/journal_article/J_Lab_Med_20031.htm

2. National Department of Health. Clinical guidelines: PMTCT (Prevention of motherto-child transmission) [document on the Internet]. c2010 [cited 2011 Apr 26] Available from: http://www.doh.gov.za/docs/factsheets/guidelines/pmtct.pdf

3. Moodley D, Esterhuizen T, Reddy L, et al. Incident HIV infection in pregnant and lactating women and its effect on mother-to-child transmission in South Africa. J Infect Dis. 2011;203(9):1231-1234. http://dx.doi.org/10.1093/infdis/jir017, PMid:21398393 
4. Inverness Medical. Determine HIV-1/2 Ag/Ab combo test package insert. In: Package insert I. Japan: Inverness Medical group, 2009; p. 1-7.

5. Guidasci T. Fourth generation point of care HIV screening. CLI Clinical Laboratory International [homepage on the Internet]. c2009 [cited 2011 Apr 28]. Available from: http://www.cli-online.com/index.php?id=2715

6. McRae B, Lange J, Ascher M, et al. Immune response to HIV p24 core protein during the early phases of human immunodeficiency virus infection. AIDS Res Hum

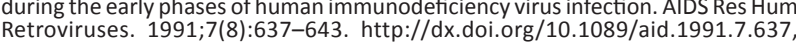
PMid:1931233

7. Beelaert G, Fransen K. Evaluation of a rapid and simple fourth-generation HIV screening assay for qualitative detection of HIV p24 antigen and/or antibodies to HIV-1 and HIV-2. J Virol Methods. 2010;168(1-2):218-222. http://dx.doi. org/10.1016/j.jviromet.2010.06.002, PMid:20561542

8. Volmink J, Sigfried NL, Merwe L, Brocklehurst P. Antiretrovirals for reducing the risk of mother-to-child transmission of HIV infection. [Cochrane review] In: The Cochrane Library, Issue 1, 2007. Oxford: Update Software. PMid:17253490

9. Department of Health. National antenatal sentinel HIV and syphilis prevalence survey in South Africa, 2009. Pretoria: National Department of Health; 2010.

10. Technau KG. Can a routine peri-partum HIV counselling and testing service for women improve access to HIV prevention, early testing and treatment of children? MSc Dissertation, Johannesburg, University of Witwatersrand, 2009.
11. Centers for Disease Control and Prevention. Guidelines for appropriate evaluations of HIV testing technologies in Africa. Department of Health and Human Services; 2001.

12. Pavie J, Rachline A, Loze B, et al., Sensitivity of five rapid HIV tests on oral fluid or finger-stick whole blood: A real-time comparison in a healthcare setting. PLoS One. 2010;5(7):e11581. http://dx.doi.org/10.1371/journal.pone.0011581, PLOS One. 2010;5(7):e11581. http:

13. World Health Organization. Guidelines for HIV diagnosis and monitoring of antiretroviral therapy: Revised version 2009. Geneva, Switzerland: WHO; 2009.

14. Tardy J. [HIV rapid testing of fourth generation]. Journee Nationale d'Infectionology Lyon [document on the Internet]. c2009 [cited 2011 Apr 27]. Available from: http://www.infectiologie.com/site/medias/JNI/JNI09/VIH/TARDY-depist-JNI09. pdf. French.

15. Schupbach J, Boni J. Quantitative and sensitive detection of immune-complexed and free HIV antigen after boiling of serum. J Virol Methods. 1993;43(2):247-256. http://dx.doi.org/10.1016/0166-0934(93)90080-B

16. Schupbach J. Measurement of HIV-1 p24 antigen by signal-amplification-boosted ELISA of heat-denatured plasma is a simple and inexpensive alternative to tests for viral RNA. AIDS Rev. 2002;4(2):83-92. PMid:12152521 